\title{
Uncut Roux-en-Y gastrojejunostomy after totally laparoscopic distal gastrectomy: Learning curve and surgical outcomes
}

\author{
Amy Kim ${ }^{1,2}$, Moon-Won Yoo \\ 'Division of Stomach Surgery, Department of Surgery, Asan Medical Center, University of Ulsan College of Medicine, Seoul; \\ ${ }^{2}$ Division of Upper Intestinal Surgery, Department of Surgery, Korea University Ansan Hospital, Ansan, Korea
}

Purpose: Totally laparoscopic distal gastrectomy (TLDG) is now widely used for early gastric cancer patients, but the selection of a reconstruction method after TLDG is still controversial. Roux-en-Y gastrojejunostomy is increasingly used in expectation of less gastritis and alkaline reflux despite its technical difficulty. The uncut Roux-en-Y gastrojejunostomy (uRYGJ) retains the advantages of Roux-en-Y reconstruction but helps prevent Roux stasis syndrome. The present study aims to introduce a single surgeon's experience of TLDG with URYGJ and analyze the learning curve and surgical outcomes.

Methods: We retrospectively reviewed the medical records of 124 consecutive patients who underwent TLDG with URYGJ performed by a single surgeon between July 2014 and August 2015 at Asan Medical Center. The baseline characteristics and surgical outcomes were analyzed, and the learning curve was drawn based on the power-law model.

Results: The mean total operative time was 165 minutes, and the average length of hospital stay was 6.6 days. Complications included two cases of duodenal stump leakage, two intra-abdominal bleeding, two intra-abdominal fluid collection, one wound problem, two anastomotic strictures, 14 ileus, and no anastomotic leakage. There were five cases of endoscopically proven reflux gastritis/esophagitis and no Roux stasis syndrome. There were five recurrences and one mortality during the follow-up period. The learning curve leveled at the 15th case.

Conclusion: The results of our study showed the safety and feasibility of URYGJ, and that the technical difficulty of the procedure can be overcome with a short learning curve for experienced surgeons.

Keywords: Stomach neoplasm, Learning curve, Gastrectomy, Laparoscopy, Roux en Y anastomosis

\section{INTRODUCTION}

Radicality and safety are the basis of surgery for malignancies [1]. Over the years, laparoscopic distal gastrectomy (LDG) has proven to be a feasible surgical option, with favorable oncologic and surgi-

Received: Jan 23, 2020 Revised: May 13, 2020 Accepted: May 27, 2020 Correspondence to: Moon-Won Yoo

Division of Stomach Surgery, Department of Surgery, Asan Medical Center, University of Ulsan College of Medicine, 88 Olympic-ro 43-gil, Songpa-gu, Seoul 05505, Korea

Tel: +82-2-3010-3484, Fax: +82-2-3010-6701

E-mail: medigang@hanmail.net

ORCID: Amy Kim (https://orcid.org/0000-0002-7021-9214), Moon-Won Yoo (https://orcid.org/0000-0003-0346-9042)

Copyright @ 2020 Korean Society of Surgical Oncology

This is an Open Access article distributed under the terms of the Creative Commons Attribution Non-Commercial License (http://creativecommons.org/licenses/by-nc/4.0) which permits unrestricted non-commercial use, distribution, and reproduction in any medium, provided the original work is properly cited. cal outcomes [2]. Since its radicality and safety have been established, more concerns are now being raised over the quality of life after surgery.

For reconstruction after LDG, Billroth I (B-I), Billroth II (B-II), and Roux-en-Y gastrojejunostomy (RYGJ) are commonly used. However, the selection of a reconstruction method after LDG still mainly depends on the surgeon's preference [3,4]. According to the Korean Gastric Cancer Association nationwide survey on gastric cancer in 2014 [5], for reconstruction after laparoscopy-assisted and totally laparoscopic distal gastrectomy (TLDG), B-I was most commonly used (51\%), followed by B-II (34.3\%) and RYGJ (9.3\%). B-I reconstruction has the advantage in that it is simpler in procedure, and results in more physiologic passage. However, it is associated with high tendency to alkaline reflux, leading to inflammation of the remnant stomach. B-II reconstruction is rarely done in Japan due to the same issue concerning bile reflux [3,4]. Despite its technical difficulty, RYGJ reconstruction is increasingly used in 
expectation of better quality of life after surgery; less gastritis and alkaline reflux [5,6]. Moreover, it is less dependent on tumor position, whereas there are some occasions B-I cannot be performed because of the expected creation of tension at the anastomosis site.

Unfortunately, Roux stasis syndrome, characterized by symptoms of food stasis in the upper gastrointestinal tract, can be complicated after RYGJ reconstruction with incidence of 5\% to 37.3\% [6-10]. The etiology of Roux stasis syndrome is still unclear, but it is thought to be caused by transection of the jejunum and separation of the Roux limb from the intestinal pacemaker $[11,12]$.

To prevent this complication, a new anastomosis method, the uncut RYGJ (uRYGJ), was designed by Van Stiegmann et al. in 1988. Zhang et al. [13] revealed advantages of the procedure in myoelectric activity and motility of the Roux limb. Several studies reported a reduced incidence of Roux stasis syndrome as well as the safety and feasibility of the procedure [14,15]. Furthermore, in 2005, Uyama et al. [16] reported a successful early surgical outcome of laparoscopy-assisted LDG with uRYGJ.

The present study aimed to introduce a single-surgeon's experience performing TLDG with uRYGJ and analyze the surgical outcomes and the learning curve.

\section{METHODS}

\section{Patient characteristics}

This study is a single-center, retrospective analysis of all consecutive patients who underwent TLDG with uRYGJ in our center between July 2014 and August 2015. All operations were performed by a single surgeon who has a previous experience of approximately 1,000 cases of gastrectomy, including 500 laparoscopic cases. The study was approved by the Institutional Review Board of Asan Medical Center (IRB No. 2018-0880). Informed consents from patients for this retrospective study were waived by institutional review board.

\section{Clinical evaluation of surgical outcomes}

We reviewed patient electronic medical charts for data collection. Baseline data included age at operation, sex, body mass index (BMI), operative time, length of hospital stay, duration until the first liquid diet, duration until first flatus, tumor size, number of retrieved and metastasized lymph nodes, distal and proximal resection margins, complications including reflux gastritis/esophagitis and Roux stasis syndrome, recurrence, and mortality. Only cases endoscopically proven were included as reflux gastritis/esophagitis.

Operation time was measured from initiation of incision to skin closure. Surgery-related complications that occurred and were detected within 30 postoperative days were defined as early compli- cations, and those that occurred after 30 days were defined as late complications.

\section{Surgical procedures}

The patient was placed in supine reverse Trendelenburg position. The first port was inserted infra-umbilically with the open Hasson technique. The other four ports were inserted with laparoscopic visualization in the upper abdomen. Mobilization of the stomach and duodenum was performed along with en-bloc lymph node dissection including partial omentectomy. The mobilized duodenum and stomach were transected with a laparoscopic linear stapler (iDrive; Covidien, North Haven, CT, USA). After retrieving the specimen, reconstruction was performed. The ligament of Treitz was exposed by retracting the transverse colon, and the jejunum at $20 \mathrm{~cm}$ distal to the ligament of Treitz was dragged up towards the stomach. Enterotomy incisions were made in the jejunum and stomach for gastrojejunostomy (GJ) using a 60-mm linear endostapler (Fig. 1A), and the common entry hole was closed with self-retaining sutures (V-Loc; Covidien) (Fig. 1B). At $45 \mathrm{~cm}$ from the GJ in the efferent loop, and at $10-15 \mathrm{~cm}$ from the ligament of Treitz in the afferent loop, enterotomy incisions were made and Braun anastomosis was performed using a 45 -mm linear endostapler (Fig. 2A), and V-loc for the common entry hole (Fig. 2B). At 2 to $3 \mathrm{~cm}$ from the GJ, a 45-mm no-knife stapler (ATS45NK: $45 \mathrm{~mm}$ ETS Articulating Linear Cutter (No Knife); Ethicon, Cincinnati, $\mathrm{OH}, \mathrm{USA}$ ) is used for the uncut procedure (Fig. 3). Closure of the jejunal mesenteric defect was routinely performed.

\section{Statistical analysis and learning curve evaluation}

SPSS 20.0 software (IBM Corp., Armonk, NY, USA) was used to analyze the characteristics and surgical outcomes of the patients. The learning curve was analyzed using Excel 2010 (Microsoft, Redmond, WA, USA) based on the power-law model.
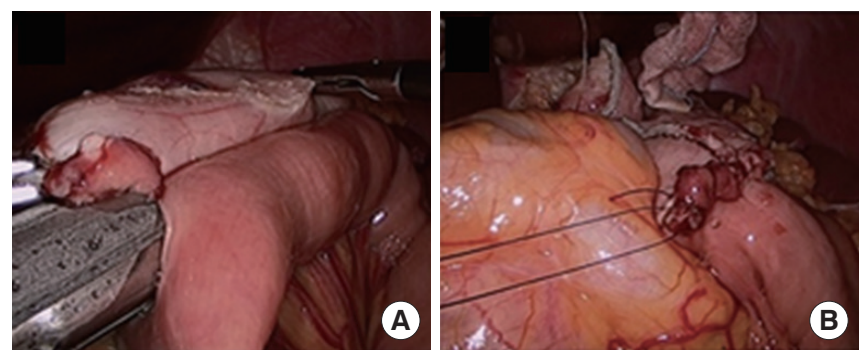

Fig. 1. Construction of gastrojejunostomy. (A) Intracorporeal isoperistaltic side-to-side gastrojejunostomy is made using $60-\mathrm{mm}$ linear stapler and (B) the common entry hole is closed with self-retaining sutures. 

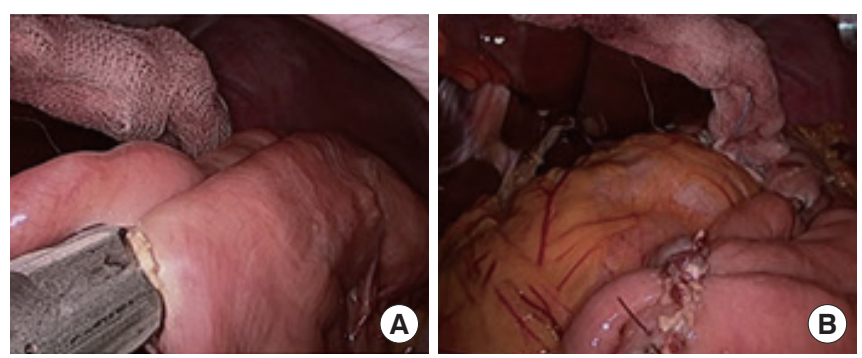

Fig. 2. Construction of jejunojejunostomy. (A) Intracorporeal Braun anastomosis is done using $45-\mathrm{mm}$ linear stapler and (B) the common entry hole is closed with self-retaining sutures.

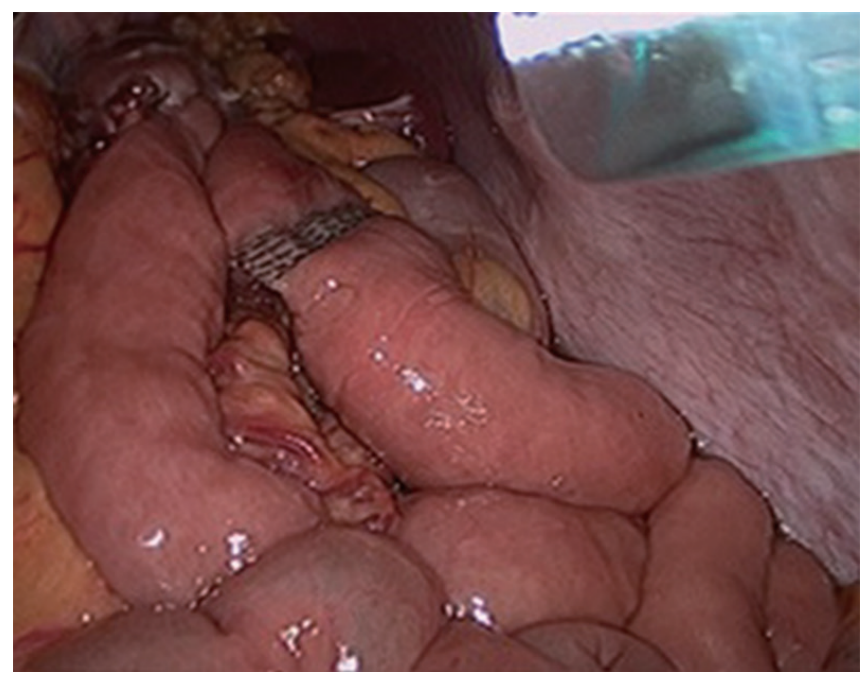

Fig. 3. Uncut procedure. A 45-mm no knife stapler is used on the afferent loop for the uncut procedure.

\section{RESULTS}

\section{Patient characteristics and surgical outcomes}

A total of 124 patients underwent TLDG with uRYGJ in our center between July 2014 and August 2015. The mean age of the patients was $57.8 \pm 11.8$ years. There were 72 male patients (58.1\%) and 52 female patients (41.9\%), and the mean BMI was $24.44 \pm 3.13$ $\mathrm{kg} / \mathrm{m}^{2}$. The median follow-up period was 38 months.

The mean total operative time was $165.4 \pm 32.5$ minutes, with variance from 107 to 285 minutes. The average length of hospital stay was $6.6 \pm 2.2$ days and liquid diet was initiated after an average of $3.4 \pm 1.1$ postoperative days. The first passage of flatus was noted after an average of $3.5 \pm 0.9$ postoperative days (Table 1 ).

The mean total number of retrieved lymph nodes and metastasized nodes in each surgery was $40.3 \pm 16.1$ and $0.3 \pm 1.1$, respectively. The average tumor size was $2.89 \pm 1.42 \mathrm{~cm}$, with a distal resection margin of $5.80 \pm 2.83 \mathrm{~cm}$ and a proximal resection margin of $4.38 \pm 2.60 \mathrm{~cm}$. Under American Joint Committee on Cancer
Table 1. Characteristics and surgical outcomes of patients who underwent TLDG with URYGJ

\begin{tabular}{lc}
\hline Characteristics and operative outcomes & Value $(n=124)$ \\
\hline Age (yr) & $57.8 \pm 11.8$ \\
Mean & $56.5(28-93)$ \\
Median & \\
Sex & $72(58.1)$ \\
Male & $52(41.9)$ \\
Female & $24.4 \pm 3.1$ \\
BMl (kg/m $\left.{ }^{2}\right)$ & $38(0-47)$ \\
Follow-up period (mo) & $165.4 \pm 32.5$ \\
Operative time (min) & \\
Operative outcomes (day) & $6.6 \pm 2.2$ \\
Length of hospital stays & $3.4 \pm 1.1$ \\
Liquid diet initiation & $3.5 \pm 0.9$ \\
Passage of flatus & $5(4.0)$ \\
Reflux gastritis/esophagitis & 0 \\
Roux stasis syndrome & $5(4.0)$ \\
Recurrence &
\end{tabular}

Values are presented as mean \pm standard deviation, median (range), or number (\%).

TLDG, totally laparoscopic distal gastrectomy; URYGJ, uncut Roux-en-Y gastrojejunostomy; BMI, body mass index.

7th edition, there were 101 patients with stage IA gastric cancer, 15 patients with stage IB, four with IIA, and one each with IIB, IIIA, IIIB, and IIIC. There were five recurrences (4\%) during the follow-up period, which included one peritoneal dissemination, two liver metastases, and two local recurrences. All patients with recurrence were stage IA at the initial surgery (Table 2).

There was no anastomotic leakage during the follow-up period. Early complications included two cases of duodenal stump leakage, two cases of intra-abdominal bleeding, four cases of ileus that delayed hospital discharge, two cases of intra-abdominal fluid collection and one wound problem. There were 11 cases of medical complications, which occurred within 30 postoperative days. These complications included temporary elevation of bilirubin or liver enzymes, pneumonia, angina, and acute myocardial infarction. Late complications included one case of gastrojejunal stricture, one case of jejunojejunal stricture, and 10 cases of mechanical ileus (Table 3).

The only mortality case resulted from myocardial infarction that occurred on the third postoperative day in a patient who did not have underlying heart disease other than hypertension. Six cases of mechanical ileus, which occurred after 30 postoperative days, resulted in re-operation, and one case of jejunojejunal stricture was managed with balloon dilatation.

There were five cases of endoscopically proven reflux gastritis/ 
Table 2. Clinicopathologic results of patients who underwent TLDG with URYGJ

\begin{tabular}{lc}
\hline Variable & Early complication \\
\hline Tumor size $(\mathrm{cm})$ & $2.89 \pm 1.42$ \\
No. of retrieved lymph nodes & $40.3 \pm 16.1$ \\
No. of metastasized lymph nodes & $0.3 \pm 1.1$ \\
Distal resection margin (cm) & $5.8 \pm 2.8$ \\
Proximal resection margin (cm) & $4.4 \pm 2.6$ \\
TNM stage & \\
IA & $101(81.5)$ \\
IB & $15(12.1)$ \\
IIA & $4(3.2)$ \\
IIB & $1(0.8)$ \\
IIIA & $1(0.8)$ \\
IIIB & $1(0.8)$ \\
IIIC & $1(0.8)$ \\
\hline
\end{tabular}

Values are presented as mean \pm standard deviation or number (\%).

TLDG, totally laparoscopic distal gastrectomy; URYGJ, uncut Roux-en-Y gastrojejunostomy.

a)TNM stages were based on the American Joint Committee on Cancer Staging Manual 7th edition.

esophagitis; and among them, one patient complained of associated symptom which involved regurgitation. There was no incidence of Roux stasis syndrome during the follow-up period (Table 1).

\section{Learning curve analysis}

The power-law model was used to evaluate the association between the number of cumulative cases and the expected operative time. With our data, the curve was best fitted at a learning rate of $95 \%$. Under this logarithmic model, the slope at a certain case number flattens as cumulative case number increases, approaching 0 . Lin et al. [17] introduced the learning curve based on the power law method and set the slope of -1 as the point in which the operator reached proficiency. We adopted this concept to identify the point at which the operator reached stability; in our study, this happened at the 15 th case.

\section{DISCUSSION}

LDG has proven its feasibility, favorable oncologic and surgical outcomes over the years [2]. However, the best reconstruction method after LDG is not yet in consensus and mainly depends on surgeon's preference. B-I, B-II, and RYGJ are commonly used reconstruction methods after LDG. B-I reconstruction is most preferred in Korea because it is technically simple and has physiological advantages [5]. However, B-I reconstruction has been associated with gastroesophageal and duodenogastric reflux.
Table 3. Surgical complication and mortality of patients who underwent TLDG with URYGJ

\begin{tabular}{lcc}
\hline Variable & Early complication & Late complication \\
\hline Complications & & 0 \\
Anastomotic leakage & 0 & 0 \\
Duodenal stump leakage & 2 & 2 \\
Anastomotic stricture & 0 & 0 \\
Pancreatic fistula & 0 & 0 \\
Bleeding & 2 & 10 \\
Mechanical ileus & 4 & 0 \\
Intra-abdominal fluid collection & 2 & 0 \\
Wound problem & 1 & 0 \\
Medical complications & 11 & 0 \\
Mortality & 1 & $-Y Y$.
\end{tabular}

TLDG, totally laparoscopic distal gastrectomy; URYGJ, uncut Roux-en-Y gastrojejunostomy.

RYGJ reconstruction is more technically demanding but supplements these disadvantages [5,6,18-20]. Also, it depends less on position of the tumor, and forms a tension-free anastomosis [21]. Some studies have even reported better food intake and nutritional benefit after surgery $[20,22]$.

Unfortunately, despite its advantages, RYGJ reconstruction is associated with Roux stasis syndrome. The etiology of this syndrome is controversial but animal studies suggest that transection of the jejunum with its ectopic pacemaker causes dysfunction of the Roux limb, leading to a slow transit of food material [8,23]. Uyama et al. developed the uRYGJ technique based on this theory and omitted the procedure of jejunal transection in order to avoid Roux stasis syndrome $[8,16]$. For the uncut procedure, instead of jejunal transection, the afferent loop is blocked with a no-knife stapler. In this way, diversion of the afferent loop is achieved without compromising the jejunal ectopic pacemaker. Performing uRYGJ after gastrectomy has proven to reduce Roux stasis syndrome $[8,21]$.

We performed a modified method of uRYGJ to accomplish a total laparoscopic surgery. A total of 124 patients received TLDG with uRYGJ at our center between July 2014 and August 2015 with a median follow-up period of 38 months. The mean total operative time was 165.4 minutes, which was comparable to other studies $[21,24]$. Reflux gastritis/esophagitis occurred in only $4.0 \%$ of the study group, and there was no incidence of Roux stasis syndrome during the follow-up period. Our results showed the safety and feasibility of uRYGJ, and that the technical difficulty of the procedure can be overcome with a relatively short learning curve for experienced surgeons. The learning curve was no longer than 10 to 29 cases for intracorporeal B-I anastomosis [25-27], which is ac- 
cepted as a technically less challenging procedure.

There are several limitations to this study. The study was based on retrospective data collection from a single surgeon, and it is not a comparative study. Another limitation is that we have no data on the recanalization of uncut stapled jejunum during the study period. It is important to evaluate recanalization because the reconstruction turns into a B-II structure when the uncut stapler line of the uRYGJ anastomosis re-opens [16,28-30]. At the time of the study, the endoscopists who performed postoperative follow-up endoscopy were not requested to observe the recanalization after uncut procedure, and there was no description regarding the re-opening on the endoscopic report. A randomized controlled trial with the establishment of a protocol for objective data collection including information on recanalization and detailed description on reflux, nutrition, and quality of life is needed to verify the functional advantage of uRYGJ compared to other anastomotic methods.

In conclusion, laparoscopic uRYGJ is feasible and safe after TLDG. The learning curve was considered to have been reached after 15 cases and this data-based evidence supports our opinion that the technical difficulty of the procedure can be overcome with a short learning curve for experienced surgeons. Therefore, uRYGJ should be considered a viable option after TLDG.

\section{CONFLICT OF INTEREST}

No potential conflict of interest relevant to this article was reported.

\section{REFERENCES}

1. Lamb P, Sivashanmugam T, White M, Irving M, Wayman J, Raimes S. Gastric cancer surgery: a balance of risk and radicality. Ann R Coll Surg Engl 2008;90:235-42.

2. Kim HH, Hyung WJ, Cho GS, Kim MC, Han SU, Kim W, et al. Morbidity and mortality of laparoscopic gastrectomy versus open gastrectomy for gastric cancer: an interim report: a phase III multicenter, prospective, randomized Trial (KLASS Trial). Ann Surg 2010;251:417-20.

3. Kim CH, Song KY, Park CH, Seo YJ, Park SM, Kim JJ. A comparison of outcomes of three reconstruction methods after laparoscopic distal gastrectomy. J Gastric Cancer 2015;15:46-52.

4. Katai H, Nunobe S, Saka M, Fukagawa T, Sano T. Reconstruction after distal gastrectomy. Nihon Geka Gakkai Zasshi 2008;109:2648.

5. Information Committee of Korean Gastric Cancer Association. Korean Gastric Cancer Association Nationwide Survey on Gastric Cancer in 2014. J Gastric Cancer 2016;16:131-40.
6. Xiong JJ, Altaf K, Javed MA, Nunes QM, Huang W, Mai G, et al. Roux-en-Y versus Billroth I reconstruction after distal gastrectomy for gastric cancer: a meta-analysis. World J Gastroenterol 2013; 19:1124-34.

7. Sun MM, Fan YY, Dang SC. Comparison between uncut Roux-en-Y and Roux-en-Y reconstruction after distal gastrectomy for gastric cancer: a meta-analysis. World J Gastroenterol 2018;24:2628-39.

8. Park YS, Shin DJ, Son SY, Kim KH, Park DJ, Ahn SH, et al. Roux stasis syndrome and gastric food stasis after laparoscopic distal gastrectomy with uncut Roux-en-Y reconstruction in gastric cancer patients: a propensity score matching analysis. World J Surg 2018;42:4022-32.

9. Obama K, Okabe H, Hosogi H, Tanaka E, Itami A, Sakai Y. Feasibility of laparoscopic gastrectomy with radical lymph node dissection for gastric cancer: from a viewpoint of pancreas-related complications. Surgery 2011;149:15-21.

10. Zang YF, Li FZ, Ji ZP, Ding YL. Application value of enhanced recovery after surgery for total laparoscopic uncut Roux-en-Y gastrojejunostomy after distal gastrectomy. World J Gastroenterol 2018;24:504-10.

11. Mathias JR, Fernandez A, Sninsky CA, Clench MH, Davis RH. Nausea, vomiting, and abdominal pain after Roux-en-Y anastomosis: motility of the jejunal limb. Gastroenterology 1985;88(1 Pt 1):101-7.

12. Miedema BW, Kelly KA. The Roux stasis syndrome: treatment by pacing and prevention by use of an 'uncut' Roux limb. Arch Surg 1992;127:295-300.

13. Zhang YM, Liu XL, Xue DB, Wei YW, Yun XG. Myoelectric activity and motility of the Roux limb after cut or uncut Roux-en-Y gastrojejunostomy. World J Gastroenterol 2006;12:7699-704.

14. Noh SM. Improvement of the Roux limb function using a new type of "uncut Roux" limb. Am J Surg 2000;180:37-40.

15. Mon RA, Cullen JJ. Standard Roux-en-Y gastrojejunostomy vs. "uncut" Roux-en-Y gastrojejunostomy: a matched cohort study. J Gastrointest Surg 2000;4:298-303.

16. Uyama I, Sakurai Y, Komori Y, Nakamura Y, Syoji M, Tonomura S, et al. Laparoscopy-assisted uncut Roux-en-Y operation after distal gastrectomy for gastric cancer. Gastric Cancer 2005;8:253-7.

17. Lin JF, Frey M, Huang JQ. Learning curve analysis of the first 100 robotic-assisted laparoscopic hysterectomies performed by a single surgeon. Int J Gynaecol Obstet 2014;124:88-91.

18. Hirao M, Takiguchi S, Imamura H, Yamamoto K, Kurokawa Y, Fujita J, et al. Comparison of Billroth I and Roux-en-Y reconstruction after distal gastrectomy for gastric cancer: one-year postoperative effects assessed by a multi-institutional RCT. Ann Surg Oncol 2013;20:1591-7.

19. Tanaka S, Matsuo K, Matsumoto H, Maki T, Nakano M, Sasaki T, et 
al. Clinical outcomes of Roux-en-Y and Billroth I reconstruction after a distal gastrectomy for gastric cancer: what is the optimal reconstructive procedure? Hepatogastroenterology 2011;58:257-62.

20. Zong L, Chen P. Billroth I vs. Billroth II vs. Roux-en-Y following distal gastrectomy: a meta-analysis based on 15 studies. Hepatogastroenterology 2011;58:1413-24.

21. Ma JJ, Zang L, Yang A, Hu WG, Feng B, Dong F, et al. A modified uncut Roux-en-Y anastomosis in totally laparoscopic distal gastrectomy: preliminary results and initial experience. Surg Endosc 2017;31:4749-55.

22. Kojima K, Yamada H, Inokuchi M, Kawano T, Sugihara K. A comparison of Roux-en-Y and Billroth-I reconstruction after laparoscopy-assisted distal gastrectomy. Ann Surg 2008;247:962-7.

23. Schirmer BD. Gastric atony and the Roux syndrome. Gastroenterol Clin North Am 1994;23:327-43.

24. Choi CI, Baek DH, Lee SH, Hwang SH, Kim DH, Kim KH, et al. Comparison between Billroth-II with Braun and Roux-en-Y reconstruction after laparoscopic distal gastrectomy. J Gastrointest Surg 2016;20:1083-90.

25. Hu GY, Tao F, Ji KW, Wang W. Comparison of delta-shape anastomosis and extracorporeal Billroth I anastomosis after laparoscopic distal gastrectomy for gastric cancer: a systematic review with me- ta-analysis of short-term outcomes. PLoS One 2016;11:e0162720.

26. Jeong O, Jung MR, Park YK, Ryu SY. Safety and feasibility during the initial learning process of intracorporeal Billroth I (delta-shaped) anastomosis for laparoscopic distal gastrectomy. Surg Endosc 2015;29:1522-9.

27. Wang B, Son SY, Shin HJ, Hur H, Han SU. The learning curve of linear-shaped gastroduodenostomy associated with totally laparoscopic distal gastrectomy. J Gastrointest Surg 2019 Jul 19 [Epub]. https://doi.org/10.1007/s11605-019-04329-3.

28. Shen Q, Yang C, Wang J, Lin M, Cai S, Li W. Application of intracorporeal uncut Roux-en- $\mathrm{Y}$ anastomosis in digestive tract reconstruction after laparoscopic total gastrectomy. Zhonghua Wei Chang Wai Ke Za Zhi 2019;22:43-8.

29. Yang L, Xu H, Zhang DC, Li FY, Wang WZ, Li Z, et al. Uncut Rouxen-Y reconstruction in a laparoscopic distal gastrectomy: a single-center study of 228 consecutive cases and short-term outcomes. Surg Innov 2019;26:698-704.

30. Yang D, He L, Tong WH, Jia ZF, Su TR, Wang Q. Randomized controlled trial of uncut Roux-en-Y vs Billroth II reconstruction after distal gastrectomy for gastric cancer: which technique is better for avoiding biliary reflux and gastritis? World J Gastroenterol 2017; 23:6350-6. 